\title{
TeV flux modulation in PSR B1259-63/LS2883
}

\author{
M. Kerschhaggl ${ }^{\star}$ \\ Department of Physics, Humboldt University, Newtonstrasse 15, Berlin, 12489, Germany \\ e-mail: mkersch@physik.hu-berlin.de
}

Received 1 March 2010 / Accepted 3 September 2010

ABSTRACT

\begin{abstract}
Context. PSR B1259-63/LS2883 is a binary system where a $48 \mathrm{~ms}$ pulsar orbits a massive Be star with a highly eccentric orbit $(e=0.87)$ with a period of 3.4 years. The system exhibits variable, non-thermal radiation visible from radio to very high energies (VHE) around periastron passage. This radiation is thought to come from particles accelerated in the shock region between the pulsar wind $(\mathrm{PW})$ and stellar outflows.

Aims. The consistency of the HESS data with the inverse Compton (IC) scenario is studied in the context of dominant orbital-phasedependent adiabatic losses, and the dependence of the observed $\mathrm{TeV}$ flux with the separation distance analysed.

Methods. Model calculations based on IC scattering of shock accelerated PW electrons and UV photons are performed. Different non-radiative cooling profiles are suggested for the primary particle population to account for the variable TeV flux.

Results. The TeV fluxes obtained with HESS in the years 2004 and 2007 only seem to depend on the binary separation. The results hint at a peculiar non-radiative cooling profile around periastron dominating the VHE emission in PSR B1259-63. The location of the stellar disc derived from this non-radiative cooling profile is in good agreement with what is inferred from radio observations.
\end{abstract}

Key words. astroparticle physics - radiation mechanisms: non-thermal - methods: numerical - binaries: general gamma rays: general - pulsars: individual: PSR B1259-63

\section{Introduction}

The binary system PSR B1259-63/LS2883 comprises a pulsar with a period of $48 \mathrm{~ms}$ and a massive star of spectral type B2Ve featuring a wind with a polar and an equatorial component (Johnston et al. 1992). The slower and denser wind in the latter constitutes a circumstellar disc. The interaction of the pulsar with the dense environment (radiation and/or matter) in the binary system may give rise to non-thermal processes. Tavani \& Arons (1997) have discussed various plausible scenarios for the production of non-thermal emission in the system. The following will focus on the non-thermal processes that occur in the shocked pulsar wind (PW) (Kennel \& Coroniti 1984). Indeed, the supersonic PW is terminated at a strong shock wave, and particles at this shock may be accelerated up to very high energies (VHE). These accelerated particles could, in the case of a leptonic PW, upscatter photons from the companion star to VHE via inverse Compton (IC) processes producing VHE emission (Tavani et al. 1994).

In the framework of this scenario, Kirk et al. (1999) have predicted the level and orbital phase dependence of the $\gamma$-ray flux. Two regimes of non-thermal particle cooling were considered: (i) negligible non-radiative (adiabatic) energy losses (i.e. dominance of radiative losses) and (ii) dominant (orbital phase independent) adiabatic losses. However, the observations of PSR B1259-63 with the HESS telescope array (Aharonian et al. 2005) have found a light curve that significantly deviates from the two light curves obtained by Kirk et al. (1999). This discrepancy supported hadronic scenarios for the production of VHE emission in the system. In the framework of this scenario, VHE protons originating in the pulsar interact with the dense outflow from the companion star, producing VHE emission.

^ Now at: Physikalisches Institut, University of Bonn, Nussallee 12, Bonn, 53115, Germany; e-mail: mkersch@physik .uni-bonn.de
Characteristic of this scenario are two sharp maxima in the VHE emission related to the pulsar passage through the dense equatorial outflow of the star (Kawachi et al. 2004). The importance of the dense circumstellar disc also seems to be indicated by the lack of pulsed radio emission shortly before and after periastron (Johnston et al. 2005). Moreover, correlations between the X-ray and $\mathrm{TeV}$ fluxes seemed to support the hadronic scenario for the VHE radiation (Chernyakova et al. 2006), since proton-proton interactions produce not only VHE $\gamma$-rays, but also secondary leptons. These secondary particles may effectively emit through the IC and synchrotron channels, providing the $\mathrm{X}$-ray and radio emission correlated with the VHE energy band (Chernyakova et al. 2006). Thus, the TeV flux from PSR B1259-63 should be related to the rate of primary interactions, i.e. to the location of the circumstellar disc on the pulsar orbit. However, it should be noted that the reconstruction of the disc properties from the eclipse of radio pulses leads to somewhat different values for the geometrical parameters of the stellar disc than those required in the hadronic scenario (Bogomazov 2005). Moreover, HESS data taken around the 2007 periastron covering orbital phases different to the ones in 2004 seem to contradict such a hadronic disc scenario since the $\mathrm{TeV}$ flux does not follow a simple disc density profile, as indicated by 2004 data (Aharonian et al. 2009). Thus, the currently obtained light curves apparently support neither leptonic nor hadronic scenarios for the production of VHE photons in PSR B1259-63. This discrepancy suggests there are additional effects to consider in the modelling. For the hadronic scenario, the simplest assumption is the orbit-to-orbit variability of the disc location. This hypothesis may be supported by the orbit-to-orbit variability of the pulsed radio emission detected from the system (Wang et al. 2004), but it seems unlikely, since there are no significant inter-orbital variations in X-ray and unpulsed radio fluxes (Chernyakova et al. 2009; Johnston et al. 2005). Clarifying this contradictory behaviour with respect to 
a hadronic disc scenario requires a dedicated study. In what follows, a possible origin of the complicated behaviour of VHE fluxes in the framework of a leptonic scenario is discussed.

The IC TeV energy flux is expected either to peak at periastron, where the target photon field density is at a maximum for the standoff point, i.e. at the location of the pulsar-star wind shock, or to show a rather smooth orbital phase dependence in the case of a saturation regime (Kirk et al. 1999); however, this contradicts what was measured by HESS. The measured VHE light curve clearly shows a drop in photon flux towards periastron. Moreover, the orbital phase dependence of the X-ray light curve (Chernyakova et al. 2006) does not fit the shape expected in a simple leptonic model (Kirk et al. 1999). All these discrepancies ask for more sophisticated modelling. Khangulyan et al. (2007) modelled the VHE data obtained with HESS in 2004 with synchrotron/IC scenarios that account for (i) a change of the acceleration rate due to enhanced losses close to the periastron passage; (ii) dominant orbital phase-dependent adiabatic losses; and (iii) the orbital phase-dependent change in the pulsar wind bulk Lorentz factor due to the interaction with stellar photons. More recently, Uchiyama et al. (2009) modelled the X-ray data obtained with the Suzaku X-ray satellite with a synchrotron and IC radiation model that included orbital phase-dependent adiabatic losses. Finally, Takata \& Taam (2009) suggest that the observed variability of non-thermal fluxes is related to the orbital phase dependence of (i) the pulsar wind bulk Lorentz factor and magnetization parameter, and (ii) the magnetization parameter and the spectral power-law index of shock-accelerated particles. In the last approach, non-radiative losses were not accounted for.

The introduction of orbital phase dependent non-radiative losses accounts for the following physical effects most likely present in PSR B1259-63: (i) the post-shock flow propagating in the confined region formed by the PW and the shocked stellar wind (Bogovalov et al. 2008) should suffer significant adiabatic losses (Khangulyan et al. 2008); (ii) the loss rate depends on the hydrodynamics of the interaction and may thus naturally depend on the orbital separation distance and the density of the stellar wind. Moreover, since the pulsar wind is expected to be anisotropic (Bogovalov \& Khangoulyan 2002), the adiabatic loss rate could have a rather complicated orbital phase dependence. We use, therefore, the approach of Khangulyan et al. (2007), taking the data obtained with HESS in 2007 into consideration.

This paper is organised as follows. First of all, a phenomenological discussion of the PSR B1259-63 light curve both in the $\gamma$-ray and X-ray bands is presented in Sect. 2. A comparison of the expected IC TeV energy flux with the HESS data allows an inference of the non-radiative cooling profile. This ansatz yields results for predicting the flux of $\mathrm{TeV}$ photons from PSR B1259-63 that describe the peculiar drop at periastron with reasonable accuracy (Khangulyan et al. 2007). In their 2007 paper, Khangulyan et al. (2007) used the HESS 2004 data to calculate the non-radiative cooling coefficients for the PSR B1259-63 orbit. In Sect. 3 of this article their analysis is done again with the addition of VHE data from the 2007 periastron passage. Moreover, three different possible cooling profiles are compared.

\section{The light curve of PSR B1259-63 as seen in X-rays and $\gamma$-rays}

While $\gamma$-ray binaries, such as LS 5039 and LSI+61303, have been observed over several orbits so could be confirmed as periodical VHE emitters (Aharonian et al. 2006; Albert et al. 2009) this has not been possible for PSR B1259-63 so far because of the rather long orbital period of 3.4 years. The overlap between the HESS datasets of 2004 and 2007 is marginal, hence inconclusive in this regard (see top panel in Fig. 1). It is thus still unknown whether the TeV light curve of this object shows periodicity. There is, however, some strong hint that the object indeed behaves periodically in the $\mathrm{TeV}$ regime: the lower panel in Fig. 1 shows the energy flux of $1 \mathrm{TeV}$ photons of PSR B1259-63 as a function of the separation distance $r$ between the pulsar and its companion star. The bidaily 2004 energy fluxes for $1 \mathrm{TeV}$ photons are taken from Aharonian et al. (2005, see their Fig. 8), whereas the 2007 fluxes are calculated from bolometric fluxes above $1 \mathrm{TeV}$ assuming the underlying spectral power-law as described in Aharonian et al. (2009). There is an apparent relation between pre and post-periastron data obtained in the years 2004 and 2007 by HESS and the orbital distance. The correlation coefficient between the 2007 fluxes shown in time bins of months and the monthly weighted mean of the bidaily 2004 data as a function of $r$ amounts to $\rho=0.89 \pm 0.12$. Despite the limited statistics of the $\mathrm{TeV}$ data and the fact that firm conclusions cannot be drawn at present, this behaviour would essentially indicate two things: (i) the TeV flux is symmetric with respect to periastron and thus mainly a function of the binary separation; (ii) the system shows indirect signs of periodicity. It should be noted that this kind of behaviour is rather unexpected in frameworks of a leptonic scenario. Indeed, since any two different orbital positions that are symmetric with respect to periastron are characterised by different IC scattering angles (according to the currently accepted orbital elements), one should expect different fluxes in the direction of the observer because of anisotropic IC scattering (Kirk et al. 1999; Aharonian \& Atoyan 1981). The significance of this effect depends on many different factors: the actual location of the production region, the $\gamma$-ray energy band, the slope of the electron spectrum, and the temperature of the optical star. Another source of asymmetry in PSR B1259-63 should be Doppler modulation of the post shock flow of relativistic particles. The orbital parameters of the system in connection with expected large Lorentz factors of the bulk flow imply a strong phase dependence of the related nonthermal emission (Khangulyan et al. 2008). Considering the system's orientation with respect to the observer (Johnston et al. 1992), this naturally should lead to an asymmetry in the observed VHE fluxes. In Fig. 2 the ratios of the VHE fluxes (integrated between $380 \mathrm{GeV}$ and $10 \mathrm{TeV}$ ) for the locations of the pulsar symmetric with respect to periastron passage (i.e. characterised by the same absolute value of the true anomaly) are shown. This figure illustrates the dependence of the flux ratio not only on the pulsar orbital location, but also on the star temperature. For the calculations, the electron distribution was assumed to be a power-law with index 2 . For the currently accepted star temperature $T_{*}=2.3 \times 10^{4} \mathrm{~K}$, the fluxes should differ by a factor of 1.5 at the locations with true anomaly $\theta \sim 2 \operatorname{rad}\left(\right.$ or $\theta \sim 115^{\circ}$, $\left.r \sim 3 \times 10^{13} \mathrm{~cm}\right)$. Apparently, the HESS observations from these epochs show almost equal fluxes (see Fig. 1) within the limited statistics of the data. In case this feature turns out to be significant, there are several possible explanations in the IC scenario for such an observational result.

(i) The ratio of the fluxes strongly depends on the temperature of the star (see Fig. 2). Indeed, if the IC scattering occurs deeper in the Klein-Nishina regime, then the cross section shows a weaker angular dependence. Thus, if the star temperature were higher by a factor of 1.5 , then the ratio of the 

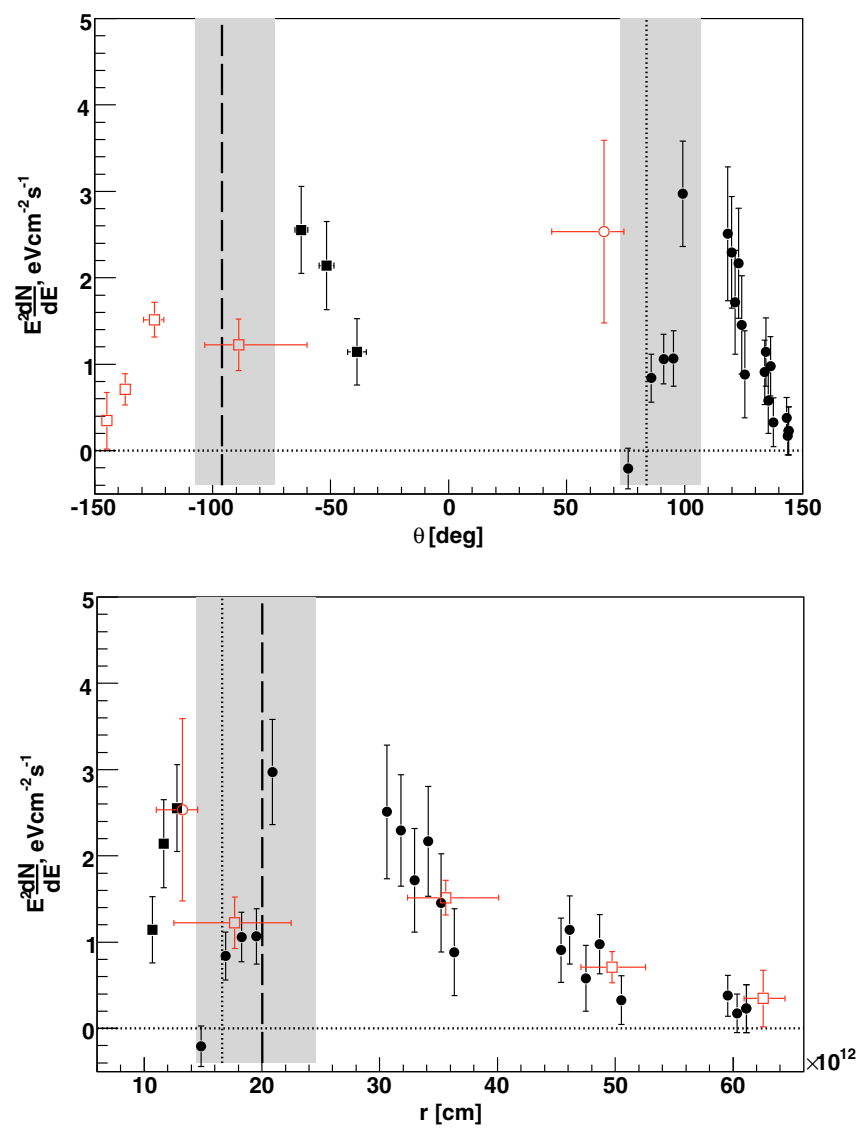

Fig. 1. Energy fluxes of $1 \mathrm{TeV}$ photons from PSR B1259-63 as a function of the true anomaly (top panel) and of the binary separation distance (bottom panel). The measurements were carried out by HESS during the 2004 (black full symbols - fluxes using a bin width of 2 days) and 2007 (red empty symbols - monthly fluxes) periastron passage, respectively. Squares denote pre- and circles post-periastron measurements. The vertical grey boxes indicate the location of the stellar disc inferred from the best-fit geometrical parameters from radio data. The vertical dashed and dotted lines denote the positions of the stellar equatorial plane along the pre- and post-periastron orbits, respectively (Bogomazov 2005).

fluxes would be consistent with the IC scenario, considering the relatively large error bars.

(ii) If the VHE emission production site has a size not much smaller than the distance to the optical star, then the IC scattering from each location of the pulsar will be characterised by different scattering angles, thus the orbital phase dependence of the VHE flux will be smoothed.

(iii) The effects of $\gamma-\gamma$ absorption, anisotropic IC scattering and Doppler boosting may compensate for each other. Thus the observed flux accidentally appears at the same level. It has to be noted, however, that this possibility requires a higher luminosity of the optical star as for photon absorption, otherwise the $\gamma-\gamma$ attenuation is negligible (Kirk et al. 1999).

While the second possibility requires a very detailed modelling of the system, which would self-consistently cover the hydrodynamics of the interaction, particle acceleration and non-thermal emission production, points (i) and (iii) (except for Doppler boosting) may be checked through a detailed study of the properties of the optical star in the system. X-ray observations can provide important information for the study of the leptonic scenario. The same electrons, which emit VHE emission through the IC scattering, will produce X-ray synchrotron emission. Moreover,

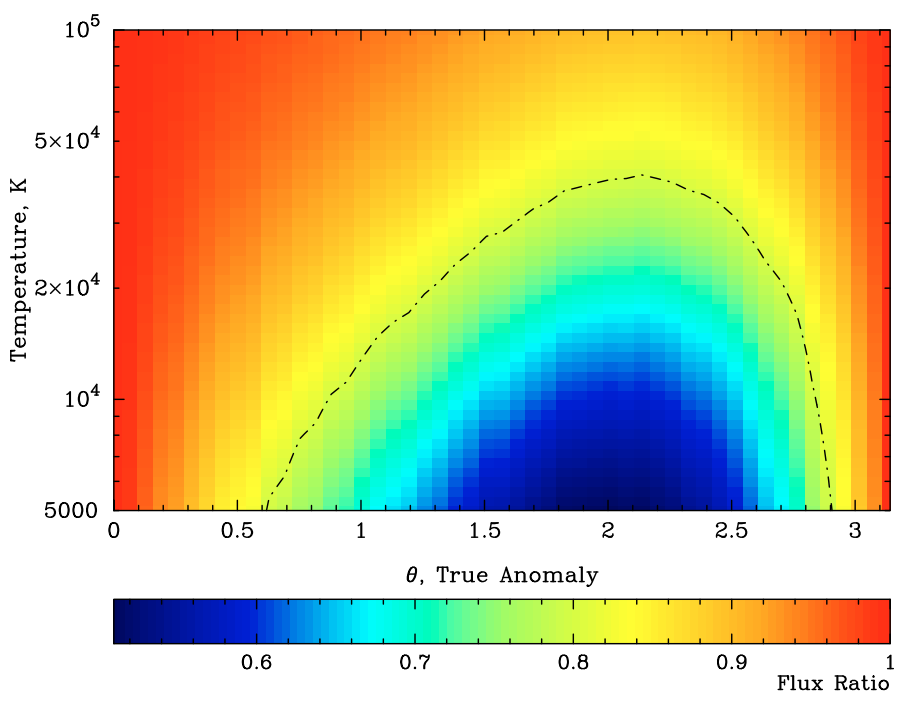

Fig. 2. Ratio of VHE fluxes (integrated between $380 \mathrm{GeV}-10 \mathrm{TeV}$ ) for any two different points on the orbit characterised by the same distance to the optical star as a function of star temperature and orbital phase. The distribution of scattering electrons was assumed to have a powerlaw index equal to 2 . The dashed-dotted line corresponds to the contour line for a flux ratio of 0.8 . Given the relatively large statistical and systematic uncertainties (close to 20\%) of the VHE data, in case of a star temperature close to (or higher than) $\sim 4 \times 10^{4} \mathrm{~K}$, the $\mathrm{TeV}$ light curve might look symmetric with respect to the periastron passage. However, if the star temperature is lower (e.g. $\sim 2 \times 10^{4} \mathrm{~K}$ ), the difference in the fluxes for orbital locations with a true anomaly of $\theta= \pm 115^{\circ}$ (or $\theta= \pm 2 \mathrm{rad}$ ) should be measurable with HESS.

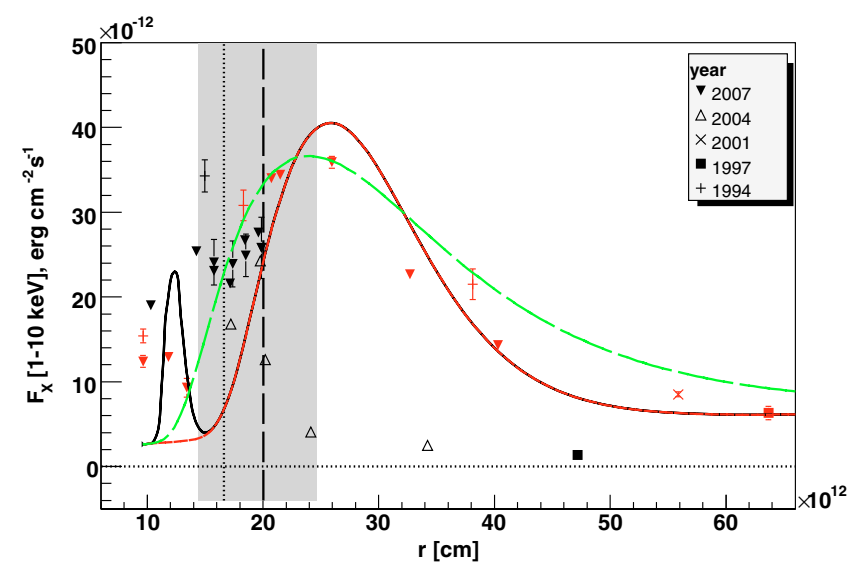

Fig. 3. X-ray fluxes in the $1-10 \mathrm{keV}$ band from pre-periastron (black symbols) and post-periastron (red symbols) phases measured in the years 1994, 1997, 2001, 2004, and 2007 as a function of the binary separation distance $r$. Also shown are three different model X-ray light curves corresponding to the adopted cooling profiles shown in Fig. 4. The grey band indicates the extension of the stellar disc. The vertical dashed and dotted lines correspond to the location of the stellar equatorial plane along the pre- and post-periastron orbit, respectively (Bogomazov 2005).

this channel has certain advantages over VHE emission: (i) $\mathrm{X}$-rays are emitted isotropically, independent of the orbital location (unless the magnetic field has an ordered structure); (ii) X-rays should not suffer absorption (given the large size of the system). However, these advantages are compensated to some extent by an additional a priori unknown parameter, the magnetic field in the production region. Considering that the magnetic field only depends on the separation distance between the two 
objects (Kirk et al. 1999; Khangulyan et al. 2007), one should expect the X-ray fluxes to be symmetric with respect to periastron passage, as is the case for $\mathrm{TeV} \gamma$-rays. In X-rays, however, such behaviour cannot be seen. In Fig. 3 X-ray data in the 1-10 keV band from ASCA (Kaspi et al. 1994; Hirayama et al. 1996), Beppo-Sax, XMM Newton (Chernyakova et al. 2006, 2009), Swift, Chandra, and Suzaku (Uchiyama et al. 2009; Chernyakova et al. 2009) from the last periastron passages since 1994 are shown as a function of the binary separation $r$. Here the picture is different from the symmetric light curve seen in the VHE regime. For $r>2 \times 10^{13} \mathrm{~cm}$ pre- and post-periastron phases are separated by almost one order of magnitude in flux since there is a steep drop in the pre-periastron part of the light curve (black symbols). Interestingly, this point of broken symmetry in the X-ray light curve coincides roughly with an increase in the TeV flux by a factor of $\sim 3$ (compare Fig. 1). The clear discrepancies between the X-ray and the $\gamma$-ray light curves presented here may indicate that the non-thermal particles responsible for the corresponding emission could be different or that the magnetic field has a rather complicated dependence on the orbital phase.

\section{Description of the model}

In this section, the model that was used to calculate the $\mathrm{TeV}$ energy flux of PSR B1259-63, taking adiabatic cooling of VHE electrons into account is briefly explained. For a more detailed description see Khangulyan et al. (2007).

The electron steady state distribution function (EDF) $n_{\mathrm{e}}(t, \gamma)$ of shock accelerated electrons as a function of time $t$ (i.e. orbital position) and energy $\gamma$ can be described by the following expression (see e.g. Ginzburg \& Syrovatskii 1964):

$n_{\mathrm{e}}(t, \gamma)=\frac{1}{|\dot{\gamma}(\gamma, t)|} \int_{\gamma}^{\infty} Q\left(\gamma^{\prime}\right) \mathrm{d} \gamma^{\prime}$.

Here, the electron energy loss rate $\dot{\gamma}$ due to e.g. synchrotron radiation (syn), IC cooling (ic), and adiabatic losses (ad) can be written as

$\dot{\gamma}=\dot{\gamma}_{\text {syn }}+\dot{\gamma}_{\text {ic }}+\dot{\gamma}_{\text {ad }}$

While the calculation of $\dot{\gamma}_{\text {syn }} \propto \mathrm{B}^{2} \gamma^{2}$ and $\dot{\gamma}_{\text {ic }}$ is straightforward (see, e.g., Bosch-Ramon \& Khangulyan 2009) it is highly not trivial to compute $\dot{\gamma}_{\text {ad }}$ analytically since it depends on the regime of the hydrodynamical interaction between the PW and the stellar outflow. Therefore, the adiabatic cooling profile is inferred by comparing an interpolation of the VHE data with the expected light curve for constant electron injection, i.e. $\dot{\gamma}_{\mathrm{ad}}=0$ (see Khangulyan et al. 2007, their Fig. 2).

The other notations in Eq. (1) are the following. The source term $Q(\gamma)$ denotes the incident injection spectrum of shocked electrons from the pulsar wind. Given the lack of understanding of the acceleration processes in binary systems, it was assumed to be a simple power-law with an energy cutoff at $E_{\mathrm{e}, \max }$ :

$Q(t, \gamma)=A \gamma^{-\alpha} \mathrm{e}^{-\frac{\gamma m c^{2}}{E_{e}, \max }} \theta\left(\gamma-\gamma_{\min }\right)$,

where $\theta$ is a Heaviside function. In general, this kind of acceleration spectrum with $\alpha \sim 2$ is consistent with observations of pulsars (e.g. Kennel \& Coroniti 1984). The spectrum is defined by the following parameters: $A$ is the normalization coefficient, determined by the fraction of PW particles transferred to VHE injection particles; $\alpha$ is the slope of the accelerated spectrum and is defined by the acceleration mechanism; and $\gamma_{\min }$

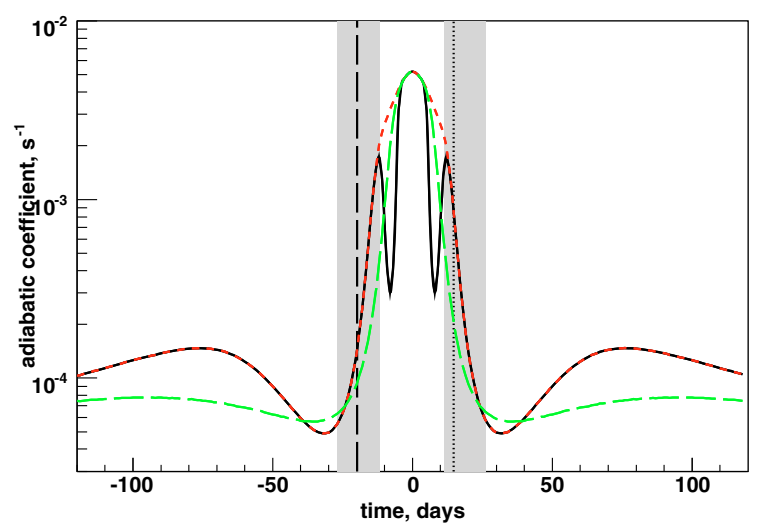

Fig. 4. Cooling coefficients for PSR B1259-63 as inferred from the combined HESS datasets shown in Fig. 1. The grey bands indicate the extension of the stellar disc. The vertical dashed and dotted lines correspond to the location of the stellar equatorial plane (Bogomazov 2005).

is the minimum energy in the accelerated spectrum. The cutoff energy $E_{\mathrm{e}, \max }$ should be determined by the balance between acceleration and losses. A simple estimate for the corresponding acceleration time scale, which is a function of the magnetic field $B(r)$, which itself depends on the binary separation $r$ as $B(r) \propto 1 / r$, is

$t_{\mathrm{acc}} \approx \eta \frac{R_{\mathrm{L}}}{c}$,

where $R_{\mathrm{L}}=m c \gamma / e B(r)$ is the Larmor radius for leptons, and $\eta>1$ characterises the acceleration rate. Comparison of Eqs. (2) and (4) provides the value of $E_{\mathrm{e}, \max }$. The EDF obtained with the inferred adiabatic loss profile was used to calculate the IC fluxes. Since adiabatic losses are assumed to be dominant, the EDF is nearly independent of the strength of the magnetic field. Attenuation of the $\mathrm{TeV}$ emission due to $\gamma-\gamma$ absorption appears to be insignificant for PSR B1259-63 (Kirk et al. 1999) so was not considered in the calculation for the VHE fluxes presented here.

\section{Results}

In this study, three different time profiles for the non-radiative cooling coefficients as shown in Fig. 4 were adopted to account for the $\gamma$-ray flux variability in PSR B1259-63 as measured by HESS. For the calculations, the following orbital parameters were used: eccentricity $e=0.87$, periastron separation $r_{0}=9.6 \times 10^{12} \mathrm{~cm}$, apastron separation $r_{\mathrm{a}}=1.4 \times 10^{14} \mathrm{~cm}$, and inclination of the orbit $i \simeq 35^{\circ}$ (Johnston et al. 1992). Using these non-radiative loss profiles for $\dot{\gamma}_{\mathrm{ad}}=\dot{\gamma}_{\mathrm{ad}}(t)$ in Eq. (2), the EDF $n_{\mathrm{e}}(t, \gamma)$ is obtained through Eq. (1). Thus, calculations of the IC energy flux using the newly computed EDFs that also account for adiabatic losses give the predictions shown in Fig. 5.

For this calculation, it was assumed that $10 \%$ of the pulsar spindown luminosity was transferred to non-thermal particles. The magnetic field at periastron was chosen to be $B=0.1 \mathrm{G}$. An energy range of $1 \mathrm{GeV}$ to $10 \mathrm{TeV}$ for the injected electron spectrum was used. Other parameters were assumed to be the same as in Khangulyan et al. (2007). In comparison, their study employed an adiabatic loss profile inferred from HESS 2004 data alone, not accounting for the drop in the VHE photon flux at period $\tau \approx 17 \mathrm{~d}$ corresponding to $\theta \approx \pm 90^{\circ}$. Uchiyama et al. (2009), on the other hand, assumed the time scale of adiabatic losses around $\tau \approx 30 \mathrm{~d}$ to be $\sim 10$ times shorter than indicated 


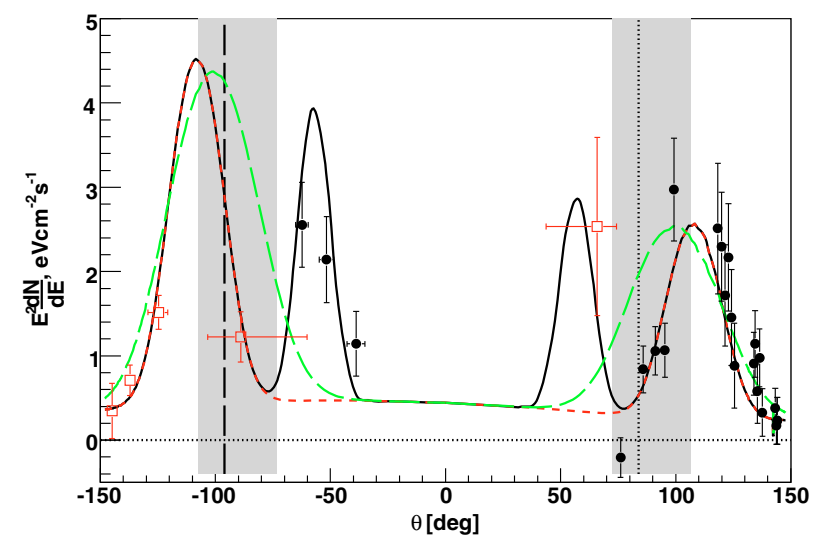

Fig. 5. Predicted energy fluxes of $1 \mathrm{TeV}$ photons from PSR B1259-63 corresponding to the three different non-radiative loss profiles as shown in Fig. 4 compared to the 2004 (black dots) and 2007 (red empty squares) HESS data. The vertical grey boxes indicate the location of the stellar disc. The vertical dashed and dotted lines denote the position of the stellar equatorial plane (Bogomazov 2005).

in this study, in order to match the broadband spectral energy distribution of the system while otherwise adopting similar parameters and radiation processes.

Comparing Figs. 1 (bottom panel) and 5 it seems that there is a peculiar "dip" in the TeV emission around $r \approx 2 \times 10^{13} \mathrm{~cm}$ that is best reproduced when assuming a non-monotonic loss rate as depicted by the black solid curve in Fig. 4. Using a monotonic and thus less complicated cooling function such as the green dash dotted profile also gives a good overall agreement with the HESS data but fails to describe the reduced photon fluxes seen at $\theta \approx \pm 90^{\circ}$.

The predictions of X-ray fluxes $(1-10 \mathrm{keV})$ for the adopted non-radiative loss profiles and model parameters are shown in Fig. 3. The pre-periastron data (black symbols) deviate considerably from the model, which adopts a symmetric behaviour of the $B$-field with respect to periastron. Obviously this assumption alone does not explain the X-ray data.

\section{Discussion and conclusions}

PSR B1259-63 has been observed for several orbits over the past decades and exhibits a globally consistent picture for the phase-dependent electromagnetic radiation at radio wavelengths, X-rays, and VHE (see e.g. Uchiyama et al. 2009, their Fig. 2). This suggests a physical link between the emission mechanisms in the different energy regimes, such as the increasing radiation efficiency with decreasing binary separation distance $r$ resulting in enhanced $\mathrm{PW}$ and stellar wind interactions. However, a more detailed comparison between the VHE and $\mathrm{X}$-ray light curves as a function of $r$ reveals substantial qualitative differences in terms of symmetry with respect to periastron and evolution in flux level. While in the X-ray light curve, Fig. 3, pre- and post-periastron data are separated by almost one order of magnitude in flux level for $r>2 \times 10^{13} \mathrm{~cm}$ such an asymmetry cannot be seen in the $\mathrm{TeV}$ band as shown in Fig. 1. Even if the $\mathrm{TeV}$ data suffers from low statistics, a similarly prominent feature in this energy regime should be notable. This is also reflected in the unexpected high TeV flux at $r \sim 3.6 \times 10^{13} \mathrm{~cm}$ $(\theta \sim-125 \mathrm{deg})$ when associating the $\mathrm{TeV}$ emission with the disc location proposed in Chernyakova et al. (2006). The corresponding disc position was inferred mainly from post-periastron data (see Aharonian et al. 2009, their Fig. 7). In this context this position suggests an asymmetric light curve due to a tilt of the stellar disc with respect to the semi minor axis of the pulsar orbit. However, it has to be noted that at present the discrepancy of the X-ray and $\mathrm{TeV}$ data in the above discussed context relies mainly on this single pre-periastron VHE point. Another deviation in the two energy bands relates to the increase in pre-periastron $\mathrm{X}$-ray fluxes seen for decreasing $r$ in the range $2.4 \times 10^{13} \mathrm{~cm}>r>1.5 \times 10^{13} \mathrm{~cm}$. This evolution appears to be reversed in the VHE light curve where there is a significant drop in the $\mathrm{TeV}$ photon flux between $r \sim 2 \times 10^{13} \mathrm{~cm}$ and $r \sim 1.5 \times 10^{13} \mathrm{~cm}$.

Considering the orbital orientation of PSR B1259-63 relative to the observer, Doppler boosting of post-shock particle flows should affect the TeV light curve and yield strong asymmetries not seen in Fig. 1. Of course Doppler boosting could always in principle be accidentally compensated for by other factors such as anisotropic IC. In general, however (and especially in case of an IC scenario), the VHE flux from a binary system is not expected to be symmetric with respect to the periastron passage. Indeed, the corresponding symmetric orbital phases are characterised by different $\gamma-\gamma$ opacities and scattering angles with regard to the line of sight. The flux symmetry with respect to periastron passage, indicated in the case of PSR B1259-63, suggests either a rather fine compensation of the above-mentioned effects or (i) a negligibly small absorption of the $\gamma$-rays and (ii) a $\gamma$-ray production mechanism, which has a weak dependence on the orbital phase. The latter could be the case for, e.g., a large production region in PSR B1259-63 or IC scattering to proceed in the deep Klein-Nishina regime with the cross section $\sigma_{\text {ic }}$ only changing marginally with $\theta$.

To explain the variability of the observed flux as a function of the separation distance, one possibility is to introduce non-radiative (adiabatic) losses, which would dominate in PSR B1259-63 over the whole orbital period (see Fig. 4 in Khangulyan et al. 2007). Moreover, the observed light curve with a number of humps and dips suggests a rather complicated dependence of the non-radiative losses on the separation distance. Given the complexity of the self-consistent calculation of the adiabatic losses, $\mathrm{TeV}$ data were used to infer a possible profile of the losses. In particular, one may expect the adiabatic loss rate to have a peak close to periastron, together with two smaller peaks located at orbital positions characterised by a true anomaly of $\theta \approx \pm 75^{\circ}$. Those smaller peaks may be linked to the impact of the stellar disc, as indicated in Fig. 4, when the pulsar exits the equatorial wind and thus the loss rate due to interference with the outflow decreases.

The predicted TeV light curves including different possible cooling profiles, Fig. 4, show an overall qualitative agreement with the data (see Fig. 5). Profiles with a simple evolution of the cooling rate, such as the dashed curves in Figs. 4 and 5, do not account for the $\mathrm{TeV}$ data close to periastron $\left(\theta \approx \pm 70^{\circ}\right)$. Naturally, the best agreement is achieved by a cooling function featuring two additional peaks that account for the potential impact of the stellar disc (black solid curve in Figs. 4 and 5). The predicted light curve shows the moderate impact of anisotropic IC scattering, while still being qualitatively compatible with the observations.

Regarding X-ray emission, the predicted light curves corresponding to the presented cooling coefficients only show a weak degree of agreement to the data (Fig. 3). The most prominent disagreement stems from pre-periastron data for binary separation distances $r>2 \times 10^{13} \mathrm{~cm}$. This basically indicates a more complicated $r$-dependence of the magnetic field in the production region than the symmetric $B(r) \propto 1 / r$ assumed in this study. There 
is qualitative agreement for the post-periastron light curve (red symbols) as far as flux level and global evolution is concerned. Introducing a second peak close to periastron in the black solid model curve seems also not justified in X-rays even if improving the prediction compared to the red dashed simple curve. In conclusion, none of the suggested model curves accounts quantitatively for the observational data in this energy regime based on a simple phase dependence of the $B$-field. This requires additional assumptions on the orbital phase dependence of the magnetic field.

Although any detailed discussion of the ratio of $\mathrm{TeV}$ and $\mathrm{X}$-ray fluxes requires fairly accurate calculations and goes beyond the scope of this paper, a qualitative explanation for a sharp increase in the X-ray flux before periastron passage may be suggested here. In the framework of dominant non-radiative losses, a decrease in the IC flux by a factor of 3 should be caused by an equivalent enhancement of the non-radiative losses. This may be achieved with an increase in the ram pressure in the stellar outflow, so that the PW termination shock moves closer to the pulsar (roughly by a factor of 3 ). Such a scenario is naturally provided by the pulsar crossing the dense stellar disc. This would not significantly change the density of the target photons (assuming the shock is located close to the pulsar), but should lead to a significant increase in the magnetic field in the production region (again by a factor of 3 ). Thus, it is indeed natural to expect an increase in the synchrotron flux by one order of magnitude. On the other hand, it has to be noted that the overall orbital behaviour of the X-ray flux cannot be explained by means of these simple arguments. In this regard it is noteworthy that the increase in pre-perisatron X-ray emission coincides with the position of the stellar disc (Fig. 3). The steepest increase in the emission is roughly aligned with the equatorial stellar plane where the disc density is presumed to be highest. Again, it is difficult to account for the behaviour of the post-periastron data in this model picture.

Although the HESS data are not yet significant enough to be conclusive regarding the existence of two dips in the TeV light curve, the symmetry of the feature presented in the VHE regime with respect to periastron and the correlation with a significant rise of the X-ray emission at the same separation distance are certainly notable. It could be explained by a non-monotonic adiabatic cooling profile, tracing a change in the shock region's size and magnetic field conditions induced by, e.g., stellar matter outflows of increased density such as the stellar disc.

Future observations in the VHE regime with instruments such as CTA or HESS II should shed light on this interesting question.

Acknowledgements. The author wants to thank F. Aharonian and D. Khangulyan for fruitful discussions and support.

\section{References}

Aharonian, F. A., \& Atoyan, A. M. 1981, Ap\&SS, 79, 321

Aharonian, F., Akhperjanian, A. G., Aye, K.-M., et al. 2005, A\&A, 442, 1

Aharonian, F., Akhperjanian, A. G., Bazer-Bachi, A. R., et al. 2006, A\&A, 460, 743

Aharonian, F., Akhperjanian, A. G., Anton, G., et al. 2009, A\&A, 507, 389

Albert, J., Aliu, E., Anderhub, H., et al. 2009, ApJ, 693, 303

Bogomazov, A. I. 2005, Astron. Rep., 49, 709

Bogovalov, S. V., \& Khangoulyan, D. V. 2002, Astron. Lett., 28, 373

Bogovalov, S. V., Khangulyan, D. V., Koldoba, A. V., Ustyugova, G. V., \& Aharonian, F. A. 2008, MNRAS, 387, 63

Bosch-Ramon, V., \& Khangulyan, D. 2009, Int. J. Mod. Phys. D, 18, 347

Chernyakova, M., Neronov, A., Lutovinov, A., Rodriguez, J., \& Johnston, S. 2006, MNRAS, 367, 1201

Chernyakova, M., Neronov, A., Aharonian, F., Uchiyama, Y., \& Takahashi, T. 2009, MNRAS, 397, 2123

Ginzburg, V., \& Syrovatskii, S. 1964, (Oxford: Pergamon Press)

Hirayama, M., Nagase, F., Tavani, M., et al. 1996, PASJ, 48, 833

Johnston, S., Manchester, R. N., Lyne, A. G., et al. 1992, ApJ, 387, L37

Johnston, S., Ball, L., Wang, N., \& Manchester, R. N. 2005, MNRAS, 358, 1069

Kaspi, V. M., Tavani, M., Nagase, F., et al. 1994, in BAAS, 26, 1485

Kawachi, A., Naito, T., Patterson, J. R., et al. 2004, ApJ, 607, 949

Kennel, C. F., \& Coroniti, F. V. 1984, ApJ, 283, 694

Khangulyan, D., Hnatic, S., Aharonian, F., \& Bogovalov, S. 2007, MNRAS, 380, 320

Khangulyan, D. V., Aharonian, F. A., Bogovalov, S. V., Koldoba, A. V., \& Ustyugova, G. V. 2008, Int. J. Mod. D, 17, 1909

Kirk, J. G., Ball, L., \& Skjaeraasen, O. 1999, Astrop. Phys., 10, 31

Takata, J., \& Taam, R. E. 2009, ApJ, 702, 100

Tavani, M., \& Arons, J. 1997, ApJ, 477, 439

Tavani, M., Arons, J., \& Kaspi, V. M. 1994, ApJ, 433, L37

Uchiyama, Y., Tanaka, T., Takahashi, T., Mori, K., \& Nakazawa, K. 2009, ApJ

Wang, N., Johnston, S., \& Manchester, R. N. 2004, MNRAS, 351, 599 